\title{
Correspondence
}

\section{Use of the child health clinic}

Sir,

We welcome the Thomas Coram Centre team's success in achieving nearly $100 \%$ attendance at their inner city clinics. ${ }^{1}$ It shows that high attendance rates can be achieved without resorting to Draconian measures. However, we challenge whether it represents an 'ideal' service, or whether frequent attendance should be synonymous with 'good'.

The team's system of 7 formal physical, developmental, and behavioural checks by a doctor before age 5 years, in addition to non-routine consultations, uses a great amount of medical time. Our experience in less wellstaffed clinics shows that such a system results in the doctor being too busy to spend sufficient time with a child with a difficult problem, and encourages the child most at risk not to attend. We doubt if repeated routine consultations reveal persistent (rather than transient) remedial abnormalities hitherto undetected; perhaps the Thomas Coram Centre has some data on this subject. In a group of 252 children (most of them under 2 years) routine examinations at an inner city clinic in Nottingham revealed only one child with an abnormality of clinical significance that had not previously been detected.

As practising clinic doctors we wonder to what extent routine checks duplicate, and therefore undermine, the work of health visitors and parents. Is the health visitor, parent, general practitioner (GP), or clinic doctor best at detecting abnormalities? In poor countries there would be no doctor available to make frequent medical checks on children; such primary care would be the responsibility of nursing staff with very basic training. In this country we deny well-trained health visitors such responsibility by insisting that checks are made by a clinic doctor. Surely primary detection is part of the role of the health visitor? We suggest that the clinic doctor or GP should have a secondary role; he should make fewer checks and look at problems identified by parents or health visitors. We are developing such a system in Nottingham.

Obviously this system will be successful only if there are sufficient staff and they are of adequate quality. Hart et al. ${ }^{1}$ make this point for doctors; we would make it equally strongly for health visitors. It is a pity that the DHSS does not have current guidelines on the patient load appropriate to health visitors. Hart et al. implied a norm of 1 health visitor for $\mathbf{2 0 0}$ families with children under 5 years, but this would not be adequate for inner cities. As the health visitor would also have older children and pensioners on her case load, we would prefer a ratio of 1 health visitor for 100 families if her role were to be enlarged to include primary screening.

Finally we are concerned that an 'ideal' service appears to exclude the GP. The role performed by Hart et al. would appear to be very similar to that performed by a GP (paediatrician), and we believe that a strong primary care service for children under 5 should integrate the GP and not bypass him.

\section{Reference}

${ }^{1}$ Hart H, Bax M, Jenkins S. Use of the child health clinic. Arch Dis Child 1981; 56: 440-5.

W Hendrickse, A Nicoll, L POLNAY, D ROBERTON, AND $S$ A SPENCER Department of Child Health, University Hospital, Queen's Medical Centre, Nottingham NG7 2UH

Dr Hart and co-workers comment:

We are grateful for the opportunity to make some slightly more polemic points than we did in our paper. We are not sure what Hendrickse et al. meant when they said that only one child with an abnormality of clinical significance was detected. We suspect that they were referring to abnormalities of those systems of the body that are morphogenically fully developed in broad terms at or around birth, and not to those in which morphogenesis continues throughout the early years of life of which the main example is the central nervous system. It is therefore not surprising that the abnormalities one detects during the early years of life are associated with development and behaviour. For example, we do not find cases of congenital heart disease as these are detected on initial investigation. Developmental and behavioural disorders are common to all ages, ${ }^{12}$ and as such problems are present in one in 5 of the population, regular assessment by a paediatrician seems reasonable to us.

We are not necessarily committed to each one of the 7 examinations we report. In fact we think that the $1 \frac{1}{2}$ to 2 years assessment is of great significance, as we begin to try to understand why so many children have problems of the development of communication. We do not want drastically to reduce the number of times we see a child nor do we believe that the proposal to ask health visitors to do developmental assessments a sensible one; as we stressed, the developing functions are assessed as part of the overall paediatric examination of the child. If the health visitor were to take on this new function her other activities would have to be curtailed.

As more is learnt about the early problems of development it is likely that the health visitor will spend more 